\title{
SUPPLEMENTARY MATERIALS: MEASURING REPRODUCIBILITY OF HIGH-THROUGHPUT EXPERIMENTS
}

By Qunhua Li, James B. Brown, Haiyan Huang, Peter J. Bickel

1. Estimation algorithm for the copula mixture model. Here we describe the iterative procedure to estimate the parameter $\theta=\left(\pi_{1}, \mu_{1}, \sigma_{1}^{2}, \rho_{1}\right)$ in detail.

1. Compute the empirical marginal CDF $\hat{F}_{j}\left(x_{i, j}\right)=\frac{r_{i, j}}{n}$ where $r_{i, j}$ is the rank of $x_{i, j}$ on replicate $j$ and $n$ is the number of pairs.

2. Rescale $\hat{F}_{j}\left(x_{i, j}\right)$ by $u_{i, j} \equiv \frac{n}{n+1} \hat{F}_{j}\left(x_{i, j}\right)$ to avoid potential unboundedness of $G^{-1}\left(u_{i, j}\right)$ if $u_{i, j}$ 's tend to one.

3. Initialize $\theta=\theta_{0}$.

4. Compute the pseudo-data $z_{i, j}=G^{-1}\left(u_{i, j} \mid \theta\right)$. As $G^{-1}$ does not have a closed form, $G$ is first computed on a grid of 1000 points for $u \in\left[\min \left(-3, \mu_{1}-3\right), \max \left(3, \mu_{1}+3\right)\right]$, then $z_{i, j}$ is obtained by linear interpolation on the grid.

5. Run EM to maximize the log-likelihood of pseudo data,

$$
l(\theta)=\sum_{i=1}^{n} \log \left(\left(1-\pi_{1}\right) h_{0}\left(z_{i, 1}, z_{i, 2}\right)+\pi_{1} h_{1}\left(z_{i, 1}, z_{i, 2} ; \mu_{1}, \sigma_{1}^{2}, \rho_{1}\right)\right)
$$

to get $\theta^{(t)}=\operatorname{argmax}_{\theta} l$. The E-step and M-step are described below.

6. Set $\theta=\theta^{(t)}$ and go to step 4 until convergence.

Here we describe the EM algorithm in step 5 above. To proceed, we denote $K_{i}$ as the latent variable, then the complete log-likelihood for the augmented pseudo data $Y_{i} \equiv\left(Z_{i}, K_{i}\right)$ is

$l^{c}=\sum_{i=1}^{n}\left[\left(1-K_{i}\right)\left(\log \left(1-\pi_{1}\right)+\log \left(h_{0}\left(z_{i, 1}, z_{i, 2}\right)\right)\right)+K_{i}\left(\log \pi_{1}+\log \left(h_{1}\left(z_{i, 1}, z_{i, 2} ; \mu_{1}, \sigma_{1}^{2}, \rho_{1}\right)\right)\right)\right]$

E-step: 


$$
\begin{aligned}
Q\left(\theta, \theta^{(t)}\right) & \equiv E\left(l^{C}(\Psi) \mid \mathbf{z}, \theta^{(t)}\right) \\
& =\sum_{i=1}^{n}\left\{P\left(K_{i}=0\right)\left[\log \left(1-\pi_{1}^{(t)}\right)+\log \left(h_{0}\left(z_{i, 1}^{(t)}, z_{i, 2}^{(t)}\right)\right)\right]\right. \\
& \left.+P\left(K_{i}=1\right)\left[\log \pi_{1}^{(t)}+\log \left(h_{1}\left(z_{i, 1}^{(t)}, z_{i, 2}^{(t)} ; \mu_{1}^{(t)},\left(\sigma_{1}^{2}\right)^{(t)}, \rho_{1}^{(t)}\right)\right)\right]\right\}
\end{aligned}
$$

Then

$$
\begin{aligned}
K_{i}^{(t+1)} & \equiv E\left(K_{i} \mid \mathbf{z}_{i}, \theta^{(t)}\right) \\
& =\frac{P\left(K_{i}=1, \mathbf{z}_{i} \mid \theta^{(t)}\right)}{P\left(\mathbf{z}_{i} \mid \theta^{(t)}\right)} \\
& =\frac{\pi_{1}^{(t)} h_{0}\left(z_{i, 1}^{(t)}, z_{i, 2}^{(t)}\right)}{\left(1-\pi_{1}^{(t)}\right) h_{0}\left(z_{i, 1}^{(t)}, z_{i, 2}^{(t)}\right)+\pi_{1}^{(t)} h_{1}\left(z_{i, 1}^{(t)}, z_{i, 2}^{(t)} ; \mu_{1}^{(t)},\left(\sigma_{1}^{2}\right)^{(t)}, \rho_{1}^{(t)}\right)}
\end{aligned}
$$

M-step:

Now we need maximize $Q\left(\theta, \theta^{(t)}\right)$. The MLE of the mixing proportion is:

$$
\pi_{1}^{(t+1)}=\frac{\sum_{i=1}^{n} K_{i}^{(t+1)}}{n}
$$

Only the 2 nd term of $Q\left(\theta, \theta^{(t)}\right)$ is relevant to $\left(\mu_{1}, \sigma_{1}^{2}, \rho_{1}\right)$, so this is equivalent to maximizing the following:

$$
\begin{aligned}
E l_{z} & =\sum_{i=1}^{n} E K_{i}\left\{\log \left(\frac{1}{2 \pi \sigma_{1}^{2} \sqrt{1-\rho_{1}^{2}}}\right)\right. \\
& \left.-\frac{1}{2\left(1-\rho_{1}^{2}\right)}\left[\frac{\left(z_{i, 1}-\mu_{1}\right)^{2}-2 \rho_{1}\left(z_{i, 1}-\mu_{1}\right)\left(z_{i, 2}-\mu_{1}\right)+\left(z_{i, 2}-\mu_{1}\right)^{2}}{\sigma_{1}^{2}}\right]\right\}
\end{aligned}
$$

Taking derivatives w.r.t. each term, we have the following:

$$
\frac{\partial l_{z}}{\partial \mu_{1}}=\sum_{i=1}^{n} \frac{K_{i}^{(t+1)}}{2\left(1-\rho_{1}^{2}\right)} \frac{2\left(z_{i, 1}-\mu_{1}\right)+2\left(z_{i, 2}-\mu_{1}\right)-2 \rho\left(z_{i, 1}+z_{i, 2}-2 \mu_{1}\right)}{\sigma_{1}^{2}}
$$

So

$$
\mu_{1}^{(t+1)}=\frac{\sum_{i=1}^{n} K_{i}^{(t+1)}\left(z_{i, 1}+z_{i, 2}\right)}{2 \sum_{i=1}^{n} K_{i}}
$$


$(1.8)$

$\frac{\partial l_{z}}{\partial \sigma_{1}^{2}}=\sum_{i=1}^{n} K^{(t+1)}\left[-\frac{1}{\sigma_{1}^{2}}+\frac{\left(z_{i, 1}-\mu_{1}\right)^{2}-2 \rho\left(z_{i, 1}-\mu_{1}\right)\left(z_{i, 2}-\mu_{1}\right)+\left(z_{i, 2}-\mu_{1}\right)^{2}}{2 \sigma_{1}^{4}\left(1-\rho_{1}^{2}\right)}\right]$

and

$$
\begin{aligned}
\frac{\partial l_{z}}{\partial \rho_{1}} & =\sum_{i=1}^{n} K^{(t+1)}\left[\frac{\rho_{1}}{1-\rho_{1}^{2}}+\frac{1}{1-\rho_{1}^{2}} \frac{\left(z_{i, 1}-\mu_{1}\right)\left(z_{i, 2}-\mu_{1}\right)}{\sigma_{1}^{2}}\right. \\
& \left.-\frac{\rho_{1}}{\left(1-\rho_{1}^{2}\right)^{2}} \frac{\left(z_{i, 1}-\mu_{1}\right)^{2}-2 \rho\left(z_{i, 1}-\mu_{1}\right)\left(z_{i, 2}-\mu_{1}\right)+\left(z_{i, 2}-\mu_{1}\right)^{2}}{\sigma_{1}^{2}}\right]
\end{aligned}
$$

Solving the above together, we get

$$
\begin{aligned}
\left(\sigma_{1}^{2}\right)^{(t+1)} & =\frac{\sum_{i=1}^{n} K_{i}\left(\left(z_{i, 1}-\mu_{1}\right)^{2}+\left(z_{i, 2}-\mu_{1}\right)^{2}\right)}{2 \sum_{i=1}^{n} K_{i}} \\
\rho_{1}^{(t+1)} & =\frac{2 \sum_{i=1}^{n} K_{i}\left(z_{i, 1}-\mu_{1}\right)\left(z_{i, 2}-\mu_{1}\right)}{\sum_{i=1}^{n} K_{i}\left[\left(z_{i, 1}-\mu_{1}\right)^{2}+\left(z_{i, 2}-\mu_{1}\right)^{2}\right]}
\end{aligned}
$$

2. Behavior of the limit of algorithm for $\boldsymbol{n}$ large. We give a heuristic argument for the asymptotic behavior of the limit of our algorithm when it converges. In another paper, we shall fill in the details of this argument. Although we are unable to prove convergence of our present algorithm with probability tending to 1 , we shall exhibit another algorithm converging with probability tending to 1 to the same limit as the present one when the latter converges.

We begin with some notation. Let $(U, V)$ be distributed according to the copula density,

$$
c(u, v, \theta)=\frac{h\left(F^{-1}(u, \theta), G^{-1}(v, \theta), \theta\right)}{f\left(F^{-1}(u, \theta)\right) g\left(G^{-1}(v, \theta)\right)}
$$

where $h(x, y, \theta)$ is a parametric family in $\theta \in \Theta \subset R^{p}$ open, $F(\cdot, \theta), G(\cdot, \theta)$ are the cdf of $X, Y$, respectively under $h(\cdot, \cdot, \theta)$, and $f(\cdot, \theta), g(\cdot, \theta)$ are the marginal densities, if $(X, Y) \sim h(\cdot, \cdot, \theta)$.

In our case, $\theta=(\epsilon, \mu, \sigma, \rho)$,

$$
h(x, y, \theta)=(1-\epsilon) \phi\left(x, y, \mu, \mu, \sigma^{2}, \sigma^{2}, \rho\right)+\epsilon \phi(x, y, 0,0,1,1,0)
$$

where $\phi\left(\cdot, \cdot, \mu, \sigma^{2}, \rho\right)$ is the density of bivariate normal with mean $\mu$, variance $\sigma^{2}$ and correlation coefficient $\rho$. 
Let $x_{i}, y_{i}, i=1, \ldots, n$ be iid with density $f$ where

$$
h(x, y) \equiv h(x, y, \theta, F, G) \equiv c(F(x), G(y), \theta) f(x) g(y),
$$

where $F, G$ are absolutely continuous marginal cdfs and $f(\cdot), g(\cdot)$ are the corresponding densities. Let $\theta_{0}$ denote the true value of $\theta$ and $F$ and $G$ be as above. Let $\hat{F}_{n}, \hat{G}_{n}$ be the empirical distribution functions of $X, Y$, respectively.

The ranks we use are $\left(\hat{F}_{n}\left(X_{i}\right), \hat{G}_{n}\left(Y_{i}\right)\right), i=1, \ldots, n$, where $\hat{F}_{n}, \hat{G}_{n}$ are the empirical cdfs of $(X, Y)$.

Our algorithm for finding $\theta$ is,

1. Initialize $\theta_{1}=\hat{\theta}_{0}$

2. Let $X_{i}(\theta) \equiv F^{-1}\left(\hat{F}_{n}\left(X_{i}\right), \theta\right)$ and $Y_{i}(\theta) \equiv G^{-1}\left(\hat{G}_{n}\left(Y_{i}\right), \theta\right)$

3. Run EM to get, assuming the initial point is close enough to the arg max below,

$$
\hat{\theta}_{1}=\operatorname{argmax}_{\theta} \int \log h(x, y, \theta) d P_{n}\left(x, y, \theta_{1}\right)
$$

where $P_{n}(x, y, \theta) \equiv \frac{1}{n} \sum_{i=1}^{n} 1\left(X_{i}(\theta) \leq x, Y_{i}(\theta) \leq y\right)$.

4. Set $\theta_{1}=\hat{\theta}_{1}$ and return to (2).

Define

$$
T_{n}(t) \equiv \operatorname{argmax}_{\theta} \int \log h(x, y, \theta) d P_{n}(x, y, t)
$$

It is clear that if our algorithm converges to $\hat{\theta}$, then $\hat{\theta}$ is a fixed point of $T_{n}$, i.e. $T_{n}(\hat{\theta})=\hat{\theta}$.

Let

$$
T(t)=\operatorname{argmax}_{\theta} \int \log h(x, y, \theta) d P(x, y, t)
$$

where $P(x, y, t)$ is the cdf of $X(t), Y(t)$. Evidently, $\theta_{0}$ is the unique fixed point of $T$ since $d P\left(x, y, \theta_{0}\right)=f\left(x, y, \theta_{0}\right) d x d y$.

Define $W_{n}(t)$ as the solution of

$$
S_{n}(\theta, t) \equiv \int \nabla_{\theta} \log h(x, y, \theta) d P_{n}(x, y, t)=0
$$

which is nearest to $t$ and a local maximum. Then, $\hat{\theta}_{1}=W_{n}\left(\hat{\theta}_{0}\right)$ and $\hat{\theta}$ is a fixed point of $W_{n}$.

Similarly, define $W(t)$ as the solution of the population version of $S_{n}$

$$
S(\theta, t) \equiv \int \nabla_{\theta} \log h(x, y, \theta) d P(x, y, t)=0
$$


which is closest to $t$ and a local maximum. Clearly $\theta_{0}$ is a fixed point of $W$.

Let $\hat{H}_{n}(\cdot, \cdot)$ be the empirical distribution function of $\left(X_{i}, Y_{i}\right)$. We can rewrite,

$$
S_{n}(\theta, t)=\int i\left(F^{-1}\left(\hat{F}_{n}(x), t\right), G^{-1}\left(\hat{G}_{n}(y), t\right), \theta\right) d \hat{H}_{n}(x, y)
$$

where $i=\nabla_{\theta} \log f$, and similarly

$$
\begin{aligned}
S(\theta, t) & =\int i\left(F^{-1}(F(x), t), G^{-1}(G(y), t), \theta\right) d H(x, y) \\
& =\int i(x, y, \theta) h(x, y, t) d x d y
\end{aligned}
$$

It is easy to see that $\theta_{0}$ is a fixed point of $W$ and is unique.

We will now study $\hat{\theta}_{n}$. We assume that

1. $\hat{\theta}_{n}$ exists

2. $\hat{\theta}_{n}$ corresponds to the unique fixed point of (2.5)

3. $\hat{\theta}_{n}$ is in $\left\{\theta:\left|\theta-\theta_{0}\right|<\epsilon\right\}$ for $\epsilon>0$ to be specified.

For simplicity, we take $p=1$ but this is inessential. As usual our point of departure is the equation

$$
-S\left(\theta_{0}, \hat{\theta}_{n}\right)=S_{n}\left(\hat{\theta}_{n}, \hat{\theta}_{n}\right)-S\left(\theta_{0}, \hat{\theta}_{n}\right)
$$

We suppose that, as we shall show, under suitable conditions elsewhere, we can expand the right hand side of (2.8) as

$$
-\frac{\partial}{\partial \theta} S\left(\theta_{0}, \theta_{0}\right)\left(\hat{\theta}_{n}-\theta_{0}\right)+O_{p}\left(\left|\hat{\theta}_{n}-\theta_{0}\right|^{2}\right)
$$

where

$$
\begin{aligned}
\left.\frac{\partial S\left(\theta_{0}, \theta_{0}\right)}{\partial t}\right|_{t=\theta_{0}} & =\left.\int i\left(x, y, \theta_{0}\right) \frac{\partial h\left(x, y, \theta_{0}\right)}{\partial t}\right|_{t=\theta_{0}} d x d y \\
& =\int i^{2}\left(x, y, \theta_{0}\right) h\left(x, y, \theta_{0}\right) d x d y
\end{aligned}
$$

We now analyze the second term in (2.8). Expand formally the integrand 
in (2.6),

$$
\begin{aligned}
& \dot{l}\left(F^{-1}\left(\hat{F}_{n}(x), \hat{\theta}_{n}\right), G^{-1}\left(\hat{G}_{n}(y), \hat{\theta}_{n}\right), \hat{\theta}_{n}\right) \\
& =i\left(F^{-1}\left(F(x), \hat{\theta}_{n}\right), G^{-1}\left(G(y), \hat{\theta}_{n}\right), \hat{\theta}_{n}\right) \\
& +\frac{1}{f\left(F^{-1}\left(F(x), \hat{\theta}_{n}\right)\right)} \frac{\partial \dot{l}}{\partial x}\left(F^{-1}\left(F(x), \hat{\theta}_{n}\right), G^{-1}\left(G(y), \hat{\theta}_{n}\right), \hat{\theta}_{n}\right)\left(\hat{F}_{n}(x)-F(x)\right) \\
& +\frac{1}{g\left(G^{-1}\left(G(y), \hat{\theta}_{n}\right)\right)} \frac{\partial \dot{l}}{\partial y}\left(F^{-1}\left(F(x), \hat{\theta}_{n}\right), G^{-1}\left(G(y), \hat{\theta}_{n}\right), \hat{\theta}_{n}\right)\left(\hat{G}_{n}(y)-G(y)\right)+O_{p}\left(n^{-1}\right)
\end{aligned}
$$

Further, formally

$$
\begin{aligned}
& \frac{1}{f\left(F^{-1}\left(F(x), \hat{\theta}_{n}\right)\right)} \frac{\partial \dot{l}}{\partial x}\left(F^{-1}\left(F(x), \hat{\theta}_{n}\right), G^{-1}\left(G(y), \hat{\theta}_{n}\right), \hat{\theta}_{n}\right)\left(\hat{F}_{n}(x)-F(x)\right) \\
& =\frac{1}{f\left(F^{-1}\left(F(x), \theta_{0}\right)\right)} \frac{\partial \dot{l}}{\partial x}\left(F^{-1}\left(F(x), \theta_{0}\right), G^{-1}\left(G(y), \theta_{0}\right), \theta_{0}\right)\left(\hat{F}_{n}(x)-F(x)\right)+O_{p}\left(\left|\hat{\theta}_{n}-\theta_{0}\right| n^{-1 / 2}\right)
\end{aligned}
$$

and a similar approximation holds for the third term in (2.10). Next note that,

$$
\begin{aligned}
& i\left(F^{-1}\left(F(x), \hat{\theta}_{n}\right), G^{-1}\left(G(y), \hat{\theta}_{n}\right), \theta_{n}\right) \\
& =i\left(F^{-1}\left(F(x), \theta_{0}\right), G^{-1}\left(G(y), \theta_{0}\right), \theta_{0}\right)+O_{p}\left(\left|\hat{\theta}_{n}-\theta_{0}\right|\right)
\end{aligned}
$$

Now expand

$$
\begin{aligned}
& \dot{l}\left(F^{-1}\left(F(x), \hat{\theta}_{n}\right), G^{-1}\left(G(y), \hat{\theta}_{n}\right), \theta_{0}\right) \\
= & \dot{l}\left(F^{-1}\left(F(x), \theta_{0}\right), G^{-1}\left(G(y), \theta_{0}\right), \theta_{0}\right)+\left.\frac{\partial \dot{l}}{\partial t}\left(F^{-1}(F(x), t), G^{-1}(G(y), t), \theta_{0}\right)\right|_{t=\theta_{0}}\left(\hat{\theta}_{n}-\theta_{0}\right) \\
& +O_{p}\left(\left|\hat{\theta}_{n}-\theta_{0}\right|^{2}\right)
\end{aligned}
$$


Combining (2.10), (2.11), (2.12), and (2.13) we get,

$$
\begin{aligned}
& S_{n}\left(\hat{\theta}_{n}, \hat{\theta}_{n}\right)-S\left(\theta_{0}, \hat{\theta}_{n}\right) \\
& =\int Q_{1}\left(x, \theta_{0}\right)\left(\hat{F}_{n}(x)-F(x)\right) d \hat{H}_{n}(x, y)+\int Q_{2}\left(y, \theta_{0}\right)\left(\hat{G}_{n}(y)-G(y)\right) d \hat{H}_{n}(x, y) \\
& +\left.\int \frac{\partial i}{\partial t}\left(F^{-1}(F(x), t), G^{-1}(G(y), t), \theta_{0}\right)\right|_{t=\theta_{0}} d H(x, y)\left(\hat{\theta}_{n}-\theta_{0}\right) \\
& +\int i\left(F^{-1}\left(F(x), \theta_{0}\right), G^{-1}\left(G(y), \theta_{0}\right), \theta_{0}\right) d\left(\hat{H}_{n}-H\right)(x, y)+O_{p}\left(n^{-1}+\left|\hat{\theta}_{n}-\theta_{0}\right|^{2}\right)
\end{aligned}
$$

where $Q_{1}\left(x, \theta_{0}\right)$ is given in (2.10) and (2.11), and similarly for $Q_{2}\left(y, \theta_{0}\right)$. All terms but the last come from the expansions (2.11), (2.13) of $i$ integrated with respect to $H$. The last comes from the difference between $S_{n}$ as given and the expression in which the measure $\hat{H}_{n}(x, y)$ is replaced by $H(x, y)$.

Combining (2.14) and (2.9), we get finally,

$M\left(\theta_{0}\right)\left(\hat{\theta}_{n}-\theta_{0}\right)=\int \dot{l}\left(x, y, \theta_{0}\right) d \hat{H}(x, y)+\int \alpha\left(x, \theta_{0}\right) d \hat{F}_{n}(x)+\int \beta\left(y, \theta_{0}\right) d \hat{G}_{n}(y)+o_{p}\left(n^{-1 / 2}\right)$

for suitable $M, \alpha, \beta$, depending on $F(\cdot), G(\cdot)$ as well as $\theta_{0}$. The heuristics yield asymptotic normality for $\hat{\theta}_{n}$. More than that $\hat{\theta}_{n}$ is asymptotically linear and its influence function is of the form $\left(\frac{\partial l}{\partial \theta}\left(x, y, \theta_{0}\right)+a\left(x, \theta_{0}, F, G\right)+\right.$ $\left.b\left(y, \theta_{0}, F, G\right)\right) M\left(\theta_{0}, F, G\right)$. By Proposition 1B (cf ? pp. 65-66) it follows that $\hat{\theta}_{n}$ is always efficient, since its influence function lies in the targent space of the model $h(\cdot, \cdot, \theta, F, G)$, where $\theta \in \Theta, F, G$ are positive densities, and $f, g$ arbitrary.

Thus in principle, these estimates are preferable to those of Genest et al (?). In a paper making these heuristics rigorous and constructing an algorithm for $\hat{\theta}_{n}$ which will converge with probability tending to 1 under suitable conditions, we will also do further extensive simulations for our Gaussian mixture and other models, comparing this procedure with that of Genest et al. We note that, in fact, no algorithm is given for the realization of their procedure except in a special case quite different from ours. We also note that our algorithm, just as that of Genest et al, depends on finding the "correct" local maximum of a multimodal function and thus depends crucially on finding appropriate starting points, in particular, running from a number of possible starting points.

\section{Derivation of properties of $\Psi$ and $\Psi^{\prime}$.}


8

Property 1: If $R\left(X_{i, 1}\right)=R\left(X_{i, 2}\right)$ for $X_{i, j} \in\left(F_{j}^{-1}(1-t), F_{j}^{-1}\left(1-t_{0}\right)\right], j=1,2$ with $0 \leq t_{0} \leq t \leq 1, \Psi(t)=\Psi\left(t_{0}\right)+t-t_{0}$ and $\Psi^{\prime}(t)=1$.

Pf:

$$
\begin{aligned}
\Psi(t) & =P\left(X_{i, 1}>F_{1}^{-1}(1-t), X_{i, 2}>F_{2}^{-1}(1-t)\right) \\
& =P\left(X_{i, 1}>F_{1}^{-1}\left(1-t_{0}\right), X_{i, 2}>F_{2}^{-1}\left(1-t_{0}\right)\right) \\
& +P\left(F_{1}^{-1}\left(1-t_{0}\right) \geq X_{i, 1}>F_{1}^{-1}(1-t), F_{2}^{-1}\left(1-t_{0}\right) \geq X_{i, 2}>F_{2}^{-1}(1-t)\right) \\
& +P\left(F_{1}^{-1}\left(1-t_{0}\right) \geq X_{i, 1}>F_{1}^{-1}(1-t), X_{i, 2}>F_{2}^{-1}\left(1-t_{0}\right)\right) \\
& +P\left(X_{i, 1}>F_{1}^{-1}\left(1-t_{0}\right), F_{2}^{-1}\left(1-t_{0}\right) \geq X_{i, 2}>F_{2}^{-1}(1-t)\right) \\
& =\Psi\left(t_{0}\right)+P\left(F_{1}^{-1}\left(1-t_{0}\right) \geq X_{i, 1}>F_{1}^{-1}(1-t)\right)+0+0 \\
& =\Psi\left(t_{0}\right)+P\left(1-t_{0} \geq F_{1}\left(X_{i, 1}\right)>1-t\right) \\
& =\Psi\left(t_{0}\right)+t-t_{0}
\end{aligned}
$$

The 3rd equation holds because $R\left(X_{i, 1}\right)=R\left(X_{i, 2}\right)$ for $X_{i, j} \in\left(F_{j}^{-1}(1-\right.$ t), $\left.F_{j}^{-1}\left(1-t_{0}\right)\right], j=1,2$. Taking derivative w.r.t. $t$, then $\Psi^{\prime}(t)=1$.

Property 2: If $R\left(X_{i, 1}\right) \perp R\left(X_{i, 2}\right)$ for $X_{i, j} \in\left(F_{j}^{-1}(1-t), F_{j}^{-1}(1)\right], j=1,2$ with $0<t \leq 1, \Psi(t)=t^{2}$ and $\Psi^{\prime}(t)=2 t$.

Pf: Since $R\left(X_{i, 1}\right) \perp R\left(X_{i, 2}\right)$ for $X_{i, j} \in\left(F_{j}^{-1}(1-t), F_{j}^{-1}(1)\right], j=1,2$,

$$
\begin{aligned}
\Psi(t) & =P\left(X_{i, 1}>F_{1}^{-1}(1-t), X_{i, 2}>F_{2}^{-1}(1-t)\right) \\
& =P\left(X_{i, 1}>F_{1}^{-1}(1-t)\right) P\left(X_{i, 2}>F_{2}^{-1}(1-t)\right)=t^{2}
\end{aligned}
$$

Taking derivative w.r.t. $t$, then $\Psi^{\prime}(t)=2 t$.

Property 3: If $R\left(X_{i, 1}\right)=R\left(X_{i, 2}\right)$ for $X_{i, j} \in\left(F_{j}^{-1}\left(1-t_{0}\right), F_{j}^{-1}(1)\right]$, and $R\left(X_{i, 1}\right) \perp$ $R\left(X_{i, 2}\right)$ for $X_{i, j} \in\left(F_{j}^{-1}(0), F_{j}^{-1}\left(1-t_{0}\right)\right], j=1,2$, where $0 \leq t_{0} \leq 1$, then for $t_{0} \leq t \leq 1, \Psi(t)=\frac{t^{2}-2 t t_{0}+t_{0}}{1-t_{0}}$ and $\Psi^{\prime}(t)=\frac{2\left(t-t_{0}\right)}{1-t_{0}}$.

Pf: If $R\left(X_{i, 1}\right)=R\left(X_{i, 2}\right)$ for $X_{i, j} \in\left(F_{j}^{-1}\left(1-t_{0}\right), F_{j}^{-1}(1)\right], \Psi\left(t_{0}\right)=t_{0}$ by Property 1. Then for $X_{i, j} \in\left(F_{j}^{-1}(t), F_{j}^{-1}\left(1-t_{0}\right)\right], j=1,2$, 


$$
\begin{aligned}
\Psi(t) & =P\left(X_{i, 1}>F_{1}^{-1}(1-t), X_{i, 2}>F_{2}^{-1}(1-t)\right) \\
& =P\left(X_{i, 1}>F_{1}^{-1}\left(1-t_{0}\right), X_{i, 2}>F_{2}^{-1}\left(1-t_{0}\right)\right) \\
& +P\left(F_{1}^{-1}\left(1-t_{0}\right) \geq X_{i, 1}>F_{1}^{-1}(1-t), F_{2}^{-1}\left(1-t_{0}\right) \geq X_{i, 2}>F_{2}^{-1}(1-t)\right) \\
& =\Psi\left(t_{0}\right)+P\left(F_{1}^{-1}\left(1-t_{0}\right) \geq X_{i, 1}>F_{1}^{-1}(1-t), F_{2}^{-1}\left(1-t_{0}\right) \geq X_{i, 2}>F_{2}^{-1}(1-t)\right. \\
& \left.\mid F_{1}^{-1}\left(1-t_{0}\right) \geq X_{i, 1}, F_{2}^{-1}\left(1-t_{0}\right) \geq X_{i, 2}\right) P\left(F_{1}^{-1}\left(1-t_{0}\right) \geq X_{i, 1}, F_{2}^{-1}\left(1-t_{0}\right) \geq X_{i, 2}\right) \\
& =t_{0}+P\left(F_{1}^{-1}\left(1-t_{0}\right) \geq X_{i, 1}>F_{1}^{-1}(1-t) \mid F_{1}^{-1}\left(1-t_{0}\right) \geq X_{i, 1}, F_{2}^{-1}\left(1-t_{0}\right) \geq X_{i, 2}\right) \\
& \times P\left(F_{2}^{-1}\left(1-t_{0}\right) \geq X_{i, 2}>F_{2}^{-1}(1-t) \mid F_{1}^{-1}\left(1-t_{0}\right) \geq X_{i, 1}, F_{2}^{-1}\left(1-t_{0}\right) \geq X_{i, 2}\right) \\
& \times P\left(F_{1}^{-1}\left(1-t_{0}\right) \geq X_{i, 1}, F_{2}^{-1}\left(1-t_{0}\right) \geq X_{i, 2}\right) \\
& =t_{0}+\frac{t-t_{0}}{1-t_{0}} \frac{t-t_{0}}{1-t_{0}}\left(1-t_{0}\right) \\
= & \frac{t^{2}-2 t t_{0}+t_{0}}{1-t_{0}}
\end{aligned}
$$

The 2nd equation holds by $R\left(X_{i, 1}\right)=R\left(X_{i, 2}\right)$ for $X_{i, j} \in\left(F_{j}^{-1}\left(t_{0}\right), F_{j}^{-1}(1)\right]$.

The 4 th equation holds because $R\left(X_{i, 1}\right) \perp R\left(X_{i, 2}\right)$ when $X_{i, j} \in\left(F_{j}^{-1}(0), F_{j}^{-1}\left(t_{0}\right)\right]$. Taking derivatice w.r.t. $t$, then $\Psi^{\prime}(t)=2 t$.

4. Extension of our model to the case of $\boldsymbol{m}>\mathbf{2}$. When $m>2$ replicates are present, the copula mixture model can be described as follows.

Let $K_{i} \sim \operatorname{Bernoulli}\left(\pi_{1}\right)$ and

$$
\left(z_{i, 1}, \ldots, z_{i, m}\right)^{T} \mid K_{i}=k \sim N\left(\left(\mu_{k}, \ldots, \mu_{k}\right)^{T},\left(\begin{array}{cccc}
\sigma_{k}^{2} & \rho_{k} \sigma_{k}^{2} & \cdots & \rho_{k} \sigma_{k}^{2} \\
\rho_{k} \sigma_{k}^{2} & \sigma_{k}^{2} & \cdots & \rho_{k} \sigma_{k}^{2} \\
\vdots & \vdots & \ddots & \vdots \\
\rho_{k} \sigma_{k}^{2} & \rho_{k} \sigma_{k}^{2} & \cdots & \sigma_{k}^{2}
\end{array}\right)\right), \quad k=0,1
$$

where $\mu_{0}=0, \mu_{1}>0, \rho_{0}=0,0<\rho_{1} \leq 1$.

Let

$$
u_{i, j} \equiv G\left(z_{i, j}\right)=\frac{\pi_{1}}{\sigma_{1}} \Phi\left(\frac{z_{i, j}-\mu_{1}}{\sigma_{1}}\right)+\pi_{0} \Phi\left(z_{i, j}\right)
$$

where $j=1, \ldots, m$. Our actual observations are

$$
x_{i, j}=F_{j}^{-1}\left(u_{i, j}\right)
$$


When $m>2$ replicates are present, the correspondence curves can be computed as follows.

$$
\Psi_{n}(t)=\frac{1}{n} \sum_{i=1}^{n} 1\left(X_{i, j} \geq x_{(\lceil(1-t) n\rceil), j}, \forall j=1, \ldots, m\right)
$$

where $m$ is the number of replicates. Then plot $t$ vs $\Psi_{n}(t)$ or $\Psi_{n}^{\prime}(t)$ similarly.

Of course, the more replicates the more stringent is this function. If the goal is to detect suboptimal samples, examining pairwise relationship using the curves presented in the manuscript for each pair is still valuable, even when there are $3+$ samples. 